\title{
Parestesia no Território do Nervo Mediano: Efeito Adverso do Estimulador de Nervo Periférico? Relato de Caso *
}

\section{Paresthesia at the Median Nerve Territory: Adverse Effect of the Peripheral Nerve Stimulator? Case Report}

\author{
Carlos Henrique Viana de Castro, TSA ${ }^{1}$, Giovanni Menezes Santos ${ }^{1}$, Niwton Carlos Toledo, TSA ${ }^{2}$,
} José Roberto de Rezende Costa, TSA ${ }^{3}$

\begin{abstract}
RESUMO
Castro CHV, Santos GM, Toledo NC, Costa JRR - Parestesia no Território do Nervo Mediano: Efeito Adverso do Estimulador de Nervo Periférico? Relato de Caso
\end{abstract}

\begin{abstract}
Justificativa e Objetivos - A Anestesiologia tem-se desenvolvido continuamente e vem sucessivamente melhorando suas técnicas, fármacos bem como seus equipamentos especialmente aqueles para monitorização. Dentre estes, o estimulador de nervo periférico destaca-se para avaliação adequada do nível de relaxamento neuromuscular e sua recuperação. Objetivou-se mostrar neste relato que, embora a monitorização moderna seja importante, por vezes, pode não ser inócua, gerando suspeição sobre sua suposta ausência de efeitos adversos.
\end{abstract}

Relato do Caso - Uma paciente de 56 anos foi submetida a cirurgia estética mamária. Seus antecedentes continham relato de diabetes mellitus, hipotireoidismo, hipertensão arterial, dislipidemia e obesidade, todos com bom controle clínico, medicamentoso e laboratorial, sem menção de complicações anestésicas em cirurgias anteriores. O procedimento transcorreu sem intercorrências e ao seu término, a paciente foi encaminhada à sala de recuperação pós-anestésica. Nesta, antes de receber alta, apresentou queixa importante de parestesia no território do nervo mediano da mão esquerda, ou seja, exatamente no local onde ficou o estimulador de nervo periférico. Tal queixa se reduziu nas próximas 48 horas, tendo a paciente recebido alta hospitalar, sem outros problemas ou alterações do exame físico.

Conclusões - A monitorização com o estimulador de nervo periférico é importante e deve ser preconizada. Para tanto, deve-se fixar e posicionar o membro adequadamente. Não obstante, esta posição pode, em alguns casos, trazer quadros neurológicos compatíveis com compressões nervosas agudas, no pós-operatório. Desta forma, deve-se atentar para diagnósticos diferenciais e, neste caso, o efeito adverso possivelmente não foi ocasionado pelo estimulador de nervo e, sim, pelo posicionamento do membro superior esquerdo.

UNITERMOS - COMPLICAÇÕES, Neurológica: parestesia; EQUIPAMENTOS: estimulador de nervo

\footnotetext{
* Recebido do (Received from) Hospital Mater Dei, Belo Horizonte, MG

1. Especialista em Terapia Intensiva e Clínica Médica

2. Legista , pós-graduado em Auditoria Médica pela Fundação Educacional Lucas Machado, Faculdade de Ciências Médicas de Minas Gerais

3. Legista , pós-graduado pelo Instituto de Ciências Biológicas da UFMG em Farmacologia Cardiovascular
}

Apresentado (Submitted) em 07 de dezembro de 2000 Aceito (Accepted) para publicação em 12 de março de 2001

Correspondência para (Mail to):

Dr. Carlos Henrique Viana de Castro

Rua do Mosteiro, 37/701 - Vila Paris

30380-780 Belo Horizonte, MG

(C) Sociedade Brasileira de Anestesiologia, 2001

\section{SUMMARY}

Castro CHV, Santos GM, Toledo NC, Costa JRR - Paresthesia at the Median Nerve Territory: Adverse Effect of the Peripheral Nerve Stimulator? Case Report

Background and Objectives - Anesthesiology is developing and improving techniques, drugs and equipment, especially monitoring devices. The peripheral nerve stimulator has been used to adequately evaluate muscle relaxation and recovery. This report aimed at showing that, although the importance of modern monitoring, it may sometimes not be innocuous, thus generating suspicion about its supposedly lack of side-effects.

Case Report - A fifty-six year-old woman was submitted to cosmetic mammaplasty. Her previous history included: diabetes mellitus, hypothyroidism, hypertension and obesity, all under good clinical control, and with no previous anesthetic complications. Surgery was performed without intercurrences and at the end patient was transferred to PACU. Before being discharged, patient complained of severe paresthesia exactly on the territory of the median nerve where the peripheral nerve stimulator had been placed. This complaint disappeared after $48 \mathrm{~h}$ and patient was discharged in good conditions.

Conclusions - Monitoring with peripheral nerve stimulator is important and should be recommended. For such, one must adequately fix and position the limb. However, such position may, in some cases, lead to neurological complications compatible with postoperative nervous compression. So, one must be alert to differential diagnosis and, in our case, it seems that the adverse effect was not caused by the nerve stimulator but by the positioning of the left upper limb.

KEY WORDS - COMPLICATIONS, Neurological: paresthesia; EQUIPMENTS: nerve stimulator

\section{INTRODUÇÃO}

A monitorização em anestesia vem apresentando contínuo desenvolvimento, com novos monitores incorporados à prática clínica rapidamente, possibilitando uma precisa leitura e interpretação dos dados clínicos do paciente. Com o estimulador de nervo periférico (ENP) não é diferente, e sua utilização transcende às salas de operação, chegando às Unidades de Terapia Intensiva (UTI), nas quais permitiu utilização mais criteriosa de bloqueadores neuromusculares (BNM), mostrando, inclusive, redução de custos e de efeitos adversos ${ }^{1,2}$. Em anestesia, a utilização do ENP se justifica pela variação da farmacocinética e farmacodinâmica dos BNM e, principalmente, para analisar a recuperação do relaxamento muscular. Recentemente, Baillard demonstrou uma incidência de $42 \%$ de TOF menor que 0,7 na Sala de Recuperação Pós-Anestésica (SRPA), mesmo usando um BNM de ação intermediária ${ }^{3}$. Apesar 
dos benefícios da monitorização moderna, deve-se atentar para os efeitos adversos de cada uma delas.

O objetivo deste artigo é relatar um caso de síndrome do túnel do carpo, no qual a parestesia no território do nervo mediano fora desencadeada pela posição da mão da paciente, supostamente em função da utilização de ENP.

\section{RELATO DO CASO}

Paciente com 56 anos de idade internou-se para colocação de prótese mamária e correção de cicatriz na mama esquerda. Sua história médica apresentava: diabetes mellitus, em uso de pioglitazona; hipotireoidismo tratado com L-tiroxina com bom controle clínico e laboratorial; obesidade; hipertensão arterial, em uso de candesartan, propranolol; dislipidemia tratada com gemfibrozil. O teste ergométrico não mostrou alterações isquêmicas e ecocardiograma revelou apenas relaxamento diastólico anormal. Demais exames hematológicos e bioquímicos sem alterações.

Antecedentes cirúrgicos: apendicectomia, amigdalectomia, histerectomia parcial e mamoplastia; sendo que as três primeiras cirurgias evoluíram sem intercorrências, e a quarta cirurgia apresentou complicação por formação de abscesso.

O exame físico revelava: PA - 110/70 mmHg; FC - 68 bpm; altura $-161 \mathrm{~cm}$; peso $84 \mathrm{~kg}$; índice de massa corporal de 32,6. $\mathrm{O}$ restante do exame físico sem alterações importantes. $\mathrm{Na}$ sala de operações recebeu midazolam (3 mg), por via venosa. Optou-se por anestesia geral, sendo feita monitorização com ECG, oximetria de pulso, capnografia, analisador de gases halogenados, estimulador de nervo periférico (ENP) no membro superior esquerdo (Figuras 1a e 1b) e pressão arterial não-invasiva no membro superior direito.

Seguiu-se a indução com propofol $\left(2 \mathrm{mg} \cdot \mathrm{kg}^{-1}\right)$, rocurônio $(0,6$ $\left.\mathrm{mg} \cdot \mathrm{kg}^{-1}\right)$, fentanil $\left(3 \mu \mathrm{g} \cdot \mathrm{kg}^{-1}\right)$ e após desaparecimento do estímulo simples, no ENP, procedeu-se a intubação traqueal com tubo número 7,5. A cirurgia transcorreu sem anormalidades, exceto pela duração de aproximadamente 4 horas e 30 minutos.
Terminada a cirurgia, a paciente acordou, foi extubada sem problemas e encaminhada à sala de recuperação pós-anestésica (SRPA), onde permaneceu por 70 minutos. Antes da alta da SRPA, queixou-se parestesia no território do nervo mediano da mão esquerda. Solicitada avaliação de um ortopedista que identificou pulsos radial e ulnar normais e teste de Phalen positivo. A parestesia reduziu progressivamente nas próximas 48 horas. A paciente recebeu alta sem queixas.

\section{DISCUSSÃO}

A síndrome do túnel do Carpo resulta da compressão do nervo mediano no túnel do carpo. Aparece na faixa etária de 30 a 60 anos, sendo cinco vezes mais freqüente em muIheres. Fatores sistêmicos associados à síndrome do túnel do carpo são: obesidade, hipotireoidismo, diabetes mellitus, amiloidose e doença de Raynaud. Em contrapartida, fatores locais que contribuem para limitação do espaço no túnel do carpo são: fratura (principalmente de Colles), edema, infecção, tumores e gânglios. Quando os sintomas aparecem durante a gravidez, freqüentemente desaparecem no puerpério. Ocasionalmente, os sintomas podem ser desencadeados por uma posição inadequada do punho durante a noite. Situação que se aproxima do caso descrito, pois uma posição viciosa por período prolongado, possivelmente, desencadeou o quadro clínico. 0 sintoma mais freqüente da síndrome do túnel do carpo é a parestesia na distribuição sensitiva do nervo mediano. O sinal de Tinel, percussão do nervo mediano no punho e a flexão do punho por 60 segundos (Teste de Phalen e Phalen invertido) podem desencadear a parestesia. Gellmane col. ${ }^{4}$ avaliaram a pressão no túnel do carpo de pacientes com síndrome do túnel do carpo na posição neutra, flexão de 90 graus e extensão de 90 graus e aferiram $32 \mathrm{mmHg}$, $99 \mathrm{mmHg}, 110 \mathrm{mmHg}$ respectivamente; enquanto que a pressão no grupo controle (sem síndrome do túnel do carpo) foi de $25 \mathrm{mmHg}$ para posição neutra, $31 \mathrm{mmHg}$ para flexão e $30 \mathrm{mmHg}$ para extensão do punho. O tratamento da
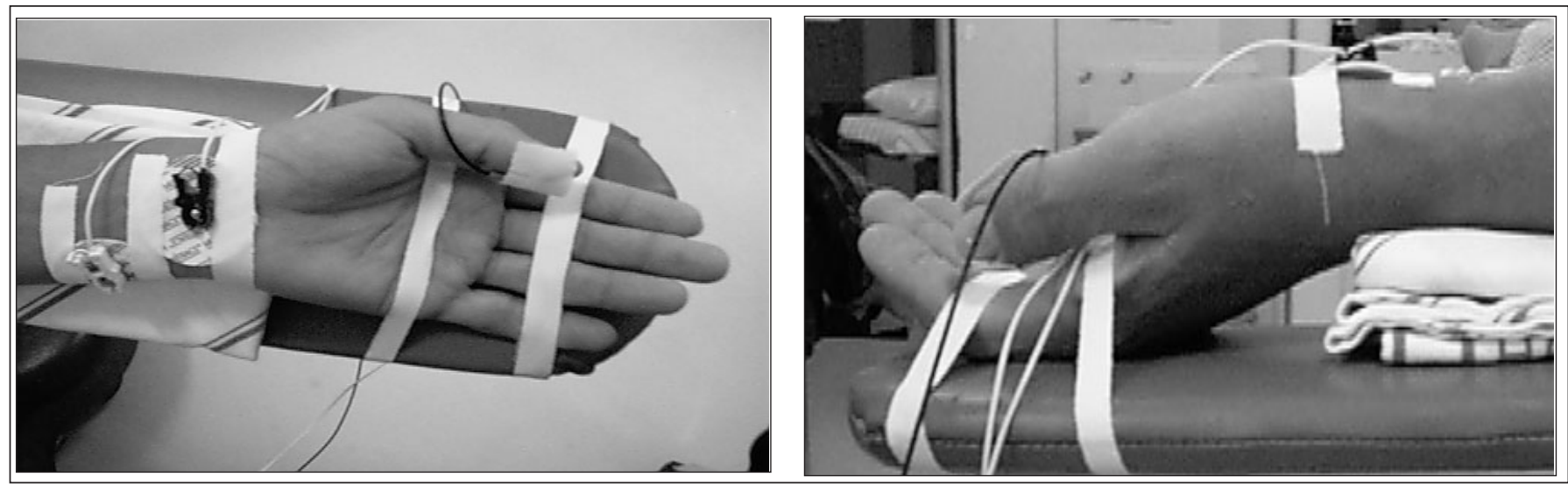

Figuras 1a e 1b - Posição e Fixação da Mão com Estimulador de Nervo Periférico 
síndrome do túnel do carpo varia com a gravidade dos sintomas, incluindo desde medicamentos ou até mesmo a descompressão cirúrgica.

O ENP é um aparelho que vem sendo usado com freqüência crescente e deve-se preconizar seu uso, quando da administração de BNM. Para uma correta interpretação dos estímulos, há necessidade de se posicionar e fixar corretamente a mão do paciente, para que movimentos de outros grupos musculares não interfiram na análise dos resultados (Figuras 1a e 1b). No entanto, esta posição viciosa pode, em pacientes susceptíveis, desencadear compressões nervosas agudas ocasionando um quadro neurológico no pós-operatório. O efeito adverso descrito, possivelmente, não teve relação direta com o estímulo elétrico do ENP, mas sim, com a posição da mão da paciente, quadro que poderia ser desencadeado mesmo sem o uso do aparelho.

\section{Paresthesia at the Median Nerve Territory: Adverse Effect of the Peripheral Nerve Stimulator? Case Report}

Carlos Henrique Viana de Castro, M.D., Giovanni Menezes Santos, M.D., Niwton Carlos Toledo, M.D., José Roberto de Rezende Costa M.D.

\section{INTRODUCTION}

Anesthesia monitoring is being continuously developed with the introduction of new monitors immediately added to the clinical practice and allowing for an accurate reading and interpretation of clinical data. This is the case of the peripheral nerve stimulator (PNS) the use of which is not confined to operating rooms but has reached Intensive Care Units (ICU) where it allowed for better neuromuscular blocker (NMB) administration, thus reducing cost and side-effects ${ }^{1,2}$. In anesthesia, the use of PNS is justified by NMB pharmacodynamic variations and, especially to analyze muscle relaxation recovery. Baillard has recently shown a $42 \%$ incidence of TOF lower than 0.7 in the Post Anesthetic Recovery Unit (PACU), even with intermediary action NMBs ${ }^{3}$. Although the benefits of modern monitoring, one must pay attention to their side-effects.

This study aimed at reporting a carpal tunnel syndrome case where paresthesia on the median nerve territory has been triggered by patient's hand positioning, supposedly as a function of PNS.

\section{CASE REPORT}

A patient 56 years of age old was admitted for mammary prosthesis and correction of left breast scar. Her medical history showed diabetes mellitus under pioglitazone; hypothyroidism treated with L-tiroxine with good clinical and laboratorial control; obesity; hypertension under candesartan and propanolol; dyslipidemia treated with gamfibrosil. Ergonomic test has not shown ischemic alterations and ECG has only revealed abnormal diastolic relaxation. Other hematological and biochemical tests were normal.

Her surgical history was: appendicectomy, tonsillectomy, partial hysterectomy and mammaplasty. The first three surgeries evolved without intercurrences and the last presented a complication by abscess formation.

Physical evaluation revealed: BP - 110/70 mmHg; HR - 68 bpm; height - $161 \mathrm{~cm}$; weight - $84 \mathrm{~kg}$; body mass index -32.6 . Remaining physical tests had no major changes. In the operating room patient was premedicated with $3 \mathrm{mg}$ intravenous midazolam and general anesthesia was induced. Monitoring consisted of ECG, pulse oximetry, capnography, halogenate gases analyzer, peripheral nerve stimulator (PNS) on the left upper limb (Figures $1 \mathrm{a}$ and $1 \mathrm{~b}$ ) and right upper limb noninvasive blood pressure.

Induction was then performed with propofol $\left(2 \mathrm{mg} \cdot \mathrm{kg}^{-1}\right)$, rocuronium $\left(0.6 \mathrm{mg}^{\mathrm{kg}}{ }^{-1}\right)$, fentanyl $\left(3 \mu \mathrm{g} . \mathrm{kg}^{-1}\right)$ and, after lack of response to simple stimulus at PNS, tracheal intubation was performed with an endotracheal tube size 7.5. Apart from lasting 4.30 hours, surgery was performed without abnormalities.
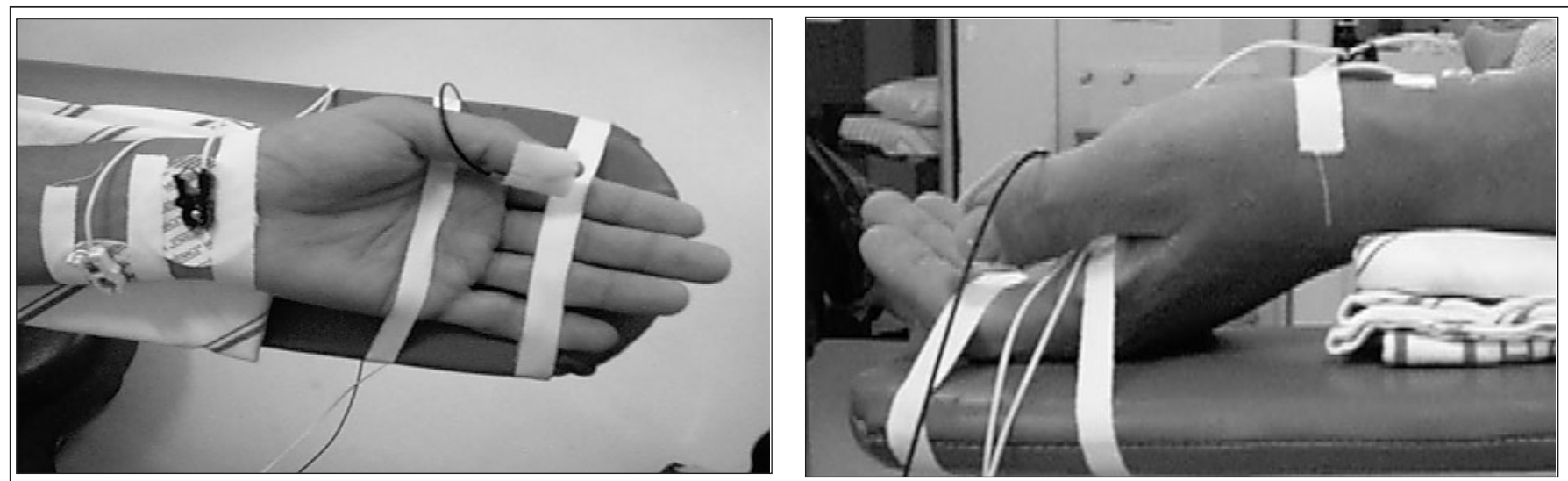

Figures $1 \mathrm{a}$ and $1 \mathrm{~b}-$ Hand Positioning and Fixation with Peripheral Nerve Stimulator 
After surgery the patient awakened, was extubated without problems and sent to the PACU where she remained for 70 minutes. Before PACU discharge, she complained of paresthesia on the left hand median nerve territory. An orthopedist was requested and identified normal radial and ulnar pulses and positive Phalen test. Paresthesia was progressively reduced along 48 hours. Patient was discharged without complaints.

\section{DIscussion}

Carpal tunnel syndrome is a consequence of the compression of the median nerve in the carpal tunnel. It appears in patients 30 to 60 years of age and is five times more frequent in women. Systemic factors associated to carpal tunnel syndrome are: obesity, hypothyroidism, diabetes mellitus, amyloidosis and Raynaud's disease. On the other hand, local factors, such as edema, infection, tumors and nodes, contribute to the limitation of the carpal tunnel space. When symptoms appear during pregnancy, they often disappear after delivery. Occasionally, symptoms may be triggered by an inadequate positioning of the fist during sleep, which is a situation close to our case report because a inadequate position for a prolonged period may have triggered the clinical event. Paresthesia in the median nerve sensory distribution is the most frequent carpal tunnel syndrome symptom. Tinel signal, median nerve percussion on the fist and fist flexion for 60 seconds (Phalen and Inverted Phalen Test) may trigger paresthesia. Gellman et al. ${ }^{4}$ have evaluated carpal tunnel pressures in patients with carpal tunnel syndrome in the neutral position -90-degree flexion and extension - and have recorded $32 \mathrm{mmHg}, 99 \mathrm{mmHg}$ and $110 \mathrm{mmHg}$, respectively, while control group pressures (without carpal tunnel syndrome) were $25 \mathrm{mmHg}$ in the neutral position, $31 \mathrm{mmHg}$ for flexion and $30 \mathrm{mmHg}$ for extension of the fist. Carpal tunnel syndrome management varies with the severity of symptoms and may range from drugs to surgical decompression.

PNS is a device which is being increasingly used and should be recommended in the presence of NMBs. To accurately interpret stimuli, patient's hands should be accurately positioned and fixed so that movements of other muscle groups will not interfere with the readings (Figure $1 \mathrm{a}$ and $1 \mathrm{~b}$ ). However, a vicious position in susceptible patients may trigger acute nervous compression leading to a postoperative neurological event. It is possible that the side-effect described had no direct relationship with PNS electrical stimulus, but being caused by the patient's hand positioning and would be triggered even without the device.

\section{REFERÊNCIAS - REFERENCES}

01. Rudis MI, Sikora CA, Angus E et al - A prospective, randomized, controlled evaluation of peripheral nerve stimulation versus standard clinical dosing of neuromuscular blocking agents in critically ill patients. Crit Care Med, 1997;25:575-583.

02. Zarowitz BJ, Rudis MI, Lai K et al - Retrospective pharmaco-e conomic evaluation of dosing vecuronium by peripheral nerve stimulation versus standard clinical assessment in critically ill patients. Pharmacotherapy, 1997;17:327-332.

03. Baillard C, Gehan G, Reboul-Marty J et al - Residual curarization the recovery room after vecuronium $\mathrm{Br}$ J Anaesth, 2000;84: 394-395.

04. Jobe MT, Wright PE - Peripheral Nerve Injuries em Cannale: Campbell's Operative Orthopaedics, $9^{\text {th }}$ Ed, Mosby, 1998; 3685-3692.

\section{RESUMEN}

Castro CHV, Santos GM, Toledo NC, Costa JRR - Parestesia en el Territorio del Nervio Mediano: Efecto Adverso del Estimulador de Nervio Periférico? Relato de Caso

Justificativa y Objetivos - La Anestesiologia se ha desenvuelto continuamente y viene sucesivamente mejorando sus técnicas, fármacos, bien como sus equipamientos, especialmente aquellos para monitorización. De entre éstos, el estimulador de nervio periférico se destaca para evaluación adecuada del nivel de relajamiento neuromuscular y su recuperación. Se objetivó mostrar en este relato que, aun cuando la monitorización moderna sea importante, por veces, puede no ser innocua, generando sospechas sobre su supuesta ausencia de efectos adversos.

Relato do Caso - Una paciente de 56 años fue sometida a cirugía estética mamaria. Sus antecedentes contenían relato de diabetes mellitus, hipotiroidismo, hipertensión arterial, dislipidemia y obesidad, todos con buen control clínico, medicamentoso y laboratorial, sin mención de complicaciones anestésicas en cirugías anteriores. El procedimiento transcurrió sin interocurrencias y a su término, la paciente fue encaminada a la sala de recuperación pós-anestésica. En ésta, antes de recibir alta, presentó queja importante de parestesia en el territorio del nervio mediano de la mano izquierda, o sea, exactamente en el local donde quedó el estimulador de nervio periférico. Tal queja se redució en las próximas 48 horas, habiendo recibido la paciente alta hospitalar, sin otros problemas o alteraciones del examen físico.

Conclusiones - La monitorización con el estimulador de nervio periférico es importante y debe ser preconizada. Para tanto, se debe fijar y posicionar el miembro adecuadamente. No obstante, esta posición puede, en algunos casos, traer cuadros neurológicos compatibles con compresiones nerviosas agudas, en el pós-operatorio. De esta forma, se debe atentar para diagnósticos diferenciales $y$, en este caso, el efecto adverso posiblemente no fue ocasionado pelo estimulador de nervio $y$, si, por el posicionamiento del miembro superior izquierdo. 